\title{
Bent necks and twisted wrists: Exploring the impact of touch-screen tablets on the posture of office workers
}

\author{
Katarzyna Stawarz and Rachel Benedyk \\ University College London \\ Gower Street, London WC1E 6BT \\ k.stawarz@cs.ucl.ac.uk, r.benedyk@ucl.ac.uk
}

\begin{abstract}
Touch-screen tablets are becoming increasingly popular as office work tools. However, as current health and safety regulations applied to office equipment do not cover tablets, the importance of understanding their impact on the posture of office workers increases. As the research on ergonomic issues related to tablet use is scarce, especially in the context of office work, we explore, through an online questionnaire, semi-structured interviews and observations with postural analysis, how and where office workers use tablets and what potential posture-related issues could arise as a result. Our findings show that the lack of screen adjustability and the virtual keyboard encourage poor posture and tablet use could lead to discomfort in a number of body areas, especially the neck and wrists.
\end{abstract}

Touch screen tablets, Posture, Office work, Ergonomics

\section{INTRODUCTION}

Touch-screen tablets (or simply 'tablets') are becoming increasingly popular: last year there were estimated 74 million tablet users in the USA alone (OPA, 2012) and one in ten UK adults (11\%) owned a tablet (Ofcom, 2012). Initially advertised as leisure and entertainment devices (Geyer \& Felske, 2011), tablets are now entering boardrooms and are increasingly used by office workers on a par with smartphones and laptops.

Yet, despite this increasing popularity, not much research has been done to evaluate tablets in the context of office work or to assess related risks. While several articles have been published over the past few years describing tablets as an office work tool (e.g. Geyer \& Felske, 2011; Nguyen \& Chaparro, 2011), and market research focusing on business use is available (e.g. Gartner, 2011), academic research is scarce. At the time of writing, only Hess and Jung (2012) and Stawarz et al. (2013) have evaluated tablets as office devices. However, their research focused on tablets' suitability for office work (Hess \& Jung) and their impact on worklife balance (Stawarz et al.), and ergonomic issues such as posture, workstation design or environment were not covered.

Even though office work, and computer use in particular, has been thoroughly researched (see Brand

(c) The Authors. Published by BCS L\&D Ltd.

Proceedings of the $27^{\text {th }}$ International BCS Human

Computer Interaction Conference
(2008) for a review of relevant research) and regulated (e.g. HSE, 2003; ISO, 2007), at present there are no health and safety regulations that directly apply to tablets. Although the UK Display Screen Equipment (DSE) Regulations (2003) cover portable devices, especially laptops, the focus is on removing portability and ensuring the device is used at a desk. ISO 9241-410 (ISO, 2007) describes input devices and covers both tablets and touchsensitive screens, but tablets using touch-screen technology are not described.

Similarly, not much has been published on risks associated with tablet use. Several health risks such as repetitive strain injury (RSI), neck pain and eyestrain have been reported in the press (e.g. Jafri, 2012; Tessler, 2012), but none of these were based on academic research. At the time of writing only Young et al. (2012; 2013) have evaluated the posture of tablet users: head and neck flexion, and wrist and shoulder posture. However, even though they covered popular tablet tasks, their studies were only conducted in a controlled lab environment and did not focus on office work 'in the wild'.

Therefore, the aim of our research was to explore the impact of tablets on office work, to understand how and why office workers use them, and to identify possible risks that could arise as a result. In this paper, we present the results of our exploratory studies and highlight possible posture issues. 


\section{OUR RESEARCH}

To better understand how tablets are used for office work and to identify possible risks, we designed an online questionnaire that explored patterns of use, the most popular tasks, and related potential issues and discomforts. We then conducted eleven semi-structured interviews to validate questionnaire findings. The interviews were supplemented with short observation sessions conducted to gather photos that were later used in postural analysis. These studies and their results are described in the following sections.

\subsection{Online questionnaire}

To understand how, when and why office workers use tablets, and what (if any) discomforts they experience, a questionnaire was designed and distributed online. "To what extent do you use your tablet for work purposes?" was used as a qualifying question and people using their tablets mainly or only for entertainment or leisure were excluded. Descriptive statistics were used for the analysis as we collected only 82 responses.

The majority of the respondents were male $(76 \%$, $\mathrm{N}=82$ ) and the average age was 30.5 years old $(S D=8.6) .38 \%$ of the respondents held managerial or business support positions, $27 \%$ had technical and $23 \%$ creative roles. $79 \%$ of the respondents used Apple products. Nearly a half $(44 \%)$ reported existing health issues such as visual impairments $(18 \%)$, back problems $(17 \%)$, and $\mathrm{RSI}(9 \%)$.

\subsubsection{Context of use and main tasks}

Over a half of the respondents (59\%) admitted using their tablet for work every day and usually on a single day they would use it for 1-2 hours (51\%). Most often office work was done at home (87\%), during the commute or while travelling ( $57 \%$ each), and in public places, e.g. cafes, pubs or airports $(50 \%)$. As a result, the most popular office tasks included checking emails (91\%), web browsing and

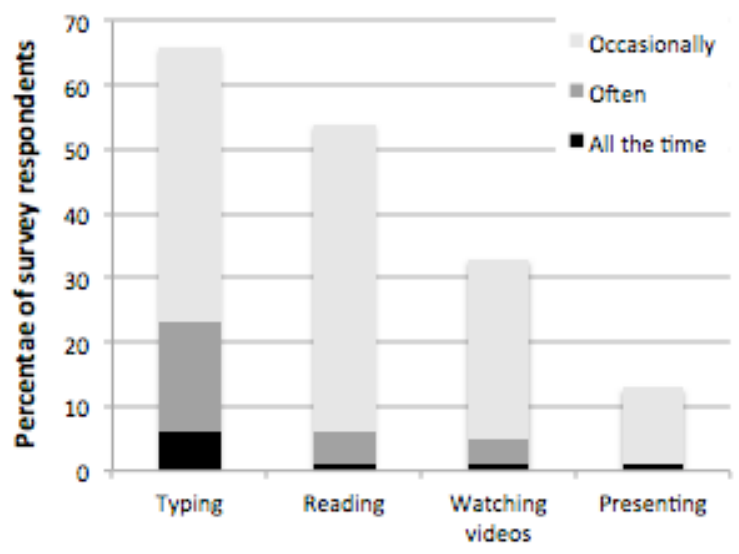

Figure 1. Percentage of survey respondents who reported experiencing discomfort while using a tablet for specific tasks

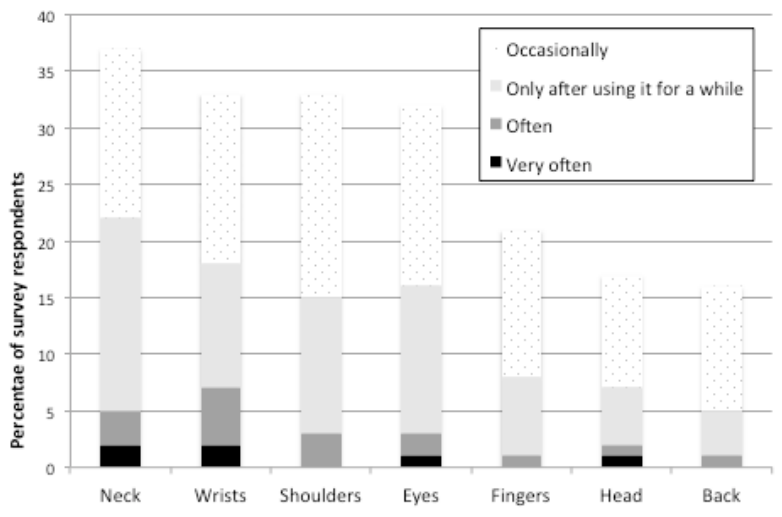

Figure 2. Percentage of survey respondents who reported experiencing discomfort in various body areas while using a tablet for work

online research $(91 \%)$, reading work documents $(79 \%)$, taking notes at meetings $(74 \%)$ and conferences $(66 \%)$, managing appointments $(70 \%)$, and reading books $(65 \%)$ - activities that can be easily done away from the office desk. In addition, $43 \%$ of the respondents mentioned using the tablet during presentations while standing up in front of the audience.

\subsubsection{Posture and discomforts}

Two thirds of the respondents $(66 \%)$ experienced discomfort at least occasionally during typing (Figure 1) and in general, discomfort or fatigue was experienced the most often in the neck, shoulders, eyes, and wrists (Figure 2). However, despite the presence of discomfort, $94 \%$ of the respondents were not aware of any tablet-related health and safety regulations within their company and only four admitted adjusting their desk to make tablet use more comfortable.

Apart from the tablet cover, which was mentioned by $85 \%$ of the respondents, tablet accessories that could help to reduce discomfort were not widely used. Some respondents used docking stations $(27 \%)$, styluses $(24 \%)$ and external keyboards $(15 \%)$, but in general tablet accessories were seen as too expensive, too cumbersome to carry and simply not needed.

\subsubsection{Discussion}

Tablets were used frequently, but for short periods, mainly for tasks that could be easily done outside an office (emails, web browsing, etc.) and users did indeed use them anywhere, especially at home or during commuting.

Because tablets were used in a variety of places and without any postural control, the fact that a large proportion of respondents reported some kind of discomfort is unsurprising, especially that some of them already suffered from back problems or RSI. This "anytime, anywhere" use also explains the lack of workstation adjustments and the reluctance towards the use of tablet accessories. 


\subsection{Interviews}

To validate the questionnaire findings and to better understand the context of use and highlighted issues and discomforts, eleven semi-structured interviews were conducted. Eight male and three female office workers aged 25-50 years old working in the media and internet industry were interviewed in their offices or in meeting rooms in their office buildings. Five worked for the same company. Six of them held managerial positions. All had been using Apple iPads for 3-12 months prior to the study and were using them mainly (although not exclusively) for work. They all had their devices with them during the interviews.

\subsubsection{Problems and discomforts}

All participants owned several portable devices: a tablet, a smartphone and a laptop, and each was used in a specific context. Tablets were used primarily during commuting and in places where laptops could not be used.

"On the way to work I use my iPad and my phone [depending how crowded the train is]. On the way home I use my iPad and my phone. At work I use my computer or my laptop, except when I go to meetings I take my iPad." - P8

Typing was the most troublesome aspect of the tablet use and the virtual keyboard was seen as inadequate for office work. The size of the keys, their response to touch, and the placement of characters were often cited as the main issues.

\section{"I find the keyboard irritating (...) I tend to miss the space bar and use V or B instead. And I insert them in the middle of the word and (..) it breaks the flow." - P4}

Some participants admitted experiencing neck and wrist pain, primarily during typing.

\begin{abstract}
"So there's a bit of neck pain involved and also I find that when I'm writing for a long time (...) in that position my wrists are slightly bent backwards and after a while (...) I feel a little bit wrist fatigue. But if I put it firmly down onto the table then also it doesn't feel right because I [have to] lift the wrists a bit and I can't see the screen as clearly as I would like to." - P11
\end{abstract}

Others mentioned difficulties with using their tablets on a crowded train, especially while standing up.

"If you're on a crowded tube, then I think it's sometimes difficult [to use the tablet] because of the size. If you don't have a seat it's difficult when you're trying to hold on to the bars." - P9

Glare and reflections were mentioned as another issue. Participants admitted that they often struggled to avoid them and had to shift the tablet, assuming awkward positions as a result.
"It doesn't work very well outside and sometimes if you have one of those overhead lights it can reflect $(. .$.$) I find myself in a meeting and I have$ to angle it." - P5

However, participants did not see discomfort and posture issues as problems that would stop them from using the device. Moreover, they were not interested in using any tablet accessories that could reduce discomfort.
"Most of my typing on the iPad is done on the tube or something, and the last thing I want to do is start carrying a keyboard, and worrying about finding something flat to use it on" - P8

\subsubsection{Discussion}

Interviews confirmed the questionnaire findings. Tablets were used in a variety of places, often as additional devices complementing laptops and smartphones. As a result, they were more likely to be used in situations where maintaining the right posture was difficult or even impossible. This use outside of the office, combined with tablets' features (their size, the lack of screen adjustability, and the virtual keyboard), encourages bad posture and could lead to neck pain and wrist discomfort.

\subsection{Observations}

Nine of the interviewees also participated in observation sessions, during which they were asked to use their tablets as normal to complete their usual tasks (e.g. compose an email, browse the web, read a work document), and were photographed while doing so. Additional wrist and finger photos were taken to support the analysis.

\subsubsection{Postural analysis}

To assess postures based on the photos, Rapid Upper Limb Assessment (RULA; McAtamney \& Corlett, 1993) was selected, as it had been previously used for laptop postural analysis (e.g. Lima \& Coelho, 2011) and therefore was deemed relevant to portable devices. Moreover, the method was specifically designed to assess sedentary tasks (Stanton et al., 2005), and all participants used

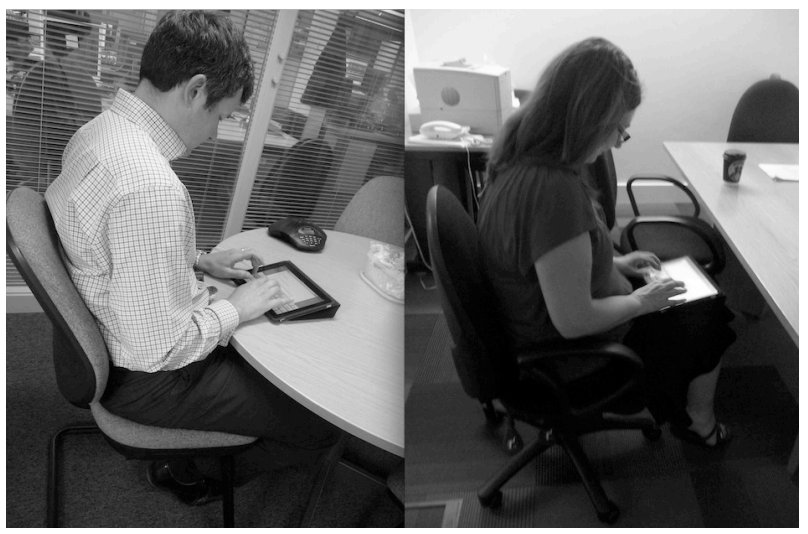

Figure 3. Research participants writing an email on their tablets 
their tablets while sitting during observations. A risk assessment checklist was created based on existing checklists and literature (UNISON, 1998; HSE, 2006; Pheasant \& Haslegrave, 2006; Oborne, $1995)$ to support RULA. Photos were analysed against the checklist and identified possible risk factors were combined with the RULA score.

The postural analysis highlighted problems with an extended neck, back, and elbow support (Figure 3). All participants exhibited bad posture: they had their necks bent and some leant forward during typing. Also, they often ignored armrests and kept their elbows in the air with their shoulders raised.

\subsubsection{Keyboard and typing analysis}

Since participants mentioned wrist discomfort and the majority of reported issues were related to the virtual keyboard, wrist angles were analysed in more detail. A series of photos was taken of a tablet used on a desk and on the lap, and with and without a cover used to adjust the angle of the keyboard (Figure 4), to analyse wrist angles and hand positions, and to assess whether the cover could reduce discomfort.

As the virtual keyboard on a tablet is narrower than a regular keyboard on a laptop or a personal computer, it caused radial hand deviation (Figure 4, top). Regardless of the location of the tablet or whether a cover was used to support typing, participants' wrists were flexed (Figure 4, middle and bottom). During breaks from typing, participants kept their fingers suspended in the air to avoid an accidental activation of the touch-screen, thus maintaining muscle tension, and giving the wrists no effective recovery time.

\subsubsection{Discussion}

Both postural and typing analyses showed that because of their flat design and the virtual keyboard, tablets encourage bad posture. While using the tablet, participants leant forward, which means that the weight of their trunk was often not properly supported, which in turn could lead to back pain. Flexed wrists and fingers suspended in the air could cause fatigue and static loading if held in such position for longer periods, and since some participants admitted typing frequently (e.g. taking notes at meetings or writing emails), this could be the main source of their discomfort.

\section{RESEARCH FINDINGS}

Combined results from our studies highlight the fact that tablets encourage bad posture and could cause discomfort. Office workers use tablets for work in various environments, mainly at home or while commuting, and since the use is not limited to a single workstation, it is difficult to ensure a good posture is maintained to minimise risks.

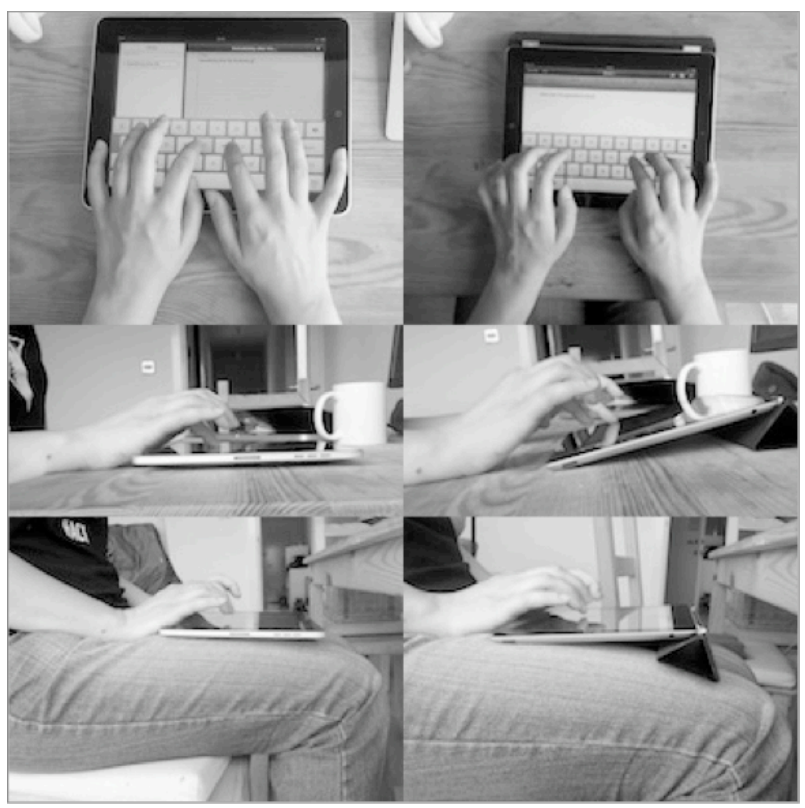

Figure 4. Wrist angles while typing on a tablet at a desk (top and middle) and on the lap (bottom), without (left) and with (right) the support of a tablet cover

Tablets are mainly used for simple tasks such as reading and responding to emails, browsing the web or reading work documents, and typing is the most popular activity. At the same time, it is also the most problematic.

The narrow virtual keyboard cannot be separated from the screen and adjusted for the comfort of use, and it lacks haptic feedback. As tablets are often kept on the lap or on low meeting room tables during typing, to see the screen users have to extend their necks and lean forward. Typing causes the wrists to be flexed and while a cover can be used to support typing, it does not prevent wrist flexion. If these positions are held for longer periods, they can increase discomfort.

Additional shoulder pain may be caused by the lack of elbow support, as users tend to ignore armrests and keep their shoulders raised when using a tablet. Some users use their tablets while standing on a train or while presenting, and therefore may experience increased manual load on the wrist to support the tablet, which could lead to further discomfort.

In addition, the glossy surface causes glare and reflection in sunlight or under overhead lighting, forcing users to tilt the tablet and change their position, which might result in assuming uncomfortable positions and lead to bad posture.

However, as tablet users often use other portable devices as well, such as laptops and smartphones, it may not be possible to point a single source of musculoskeletal issues, especially that some users already suffer from back pain and RSI. 


\section{DISCUSSION AND FUTURE WORK}

The goal of our research was to understand how and why office workers use tablets, and to explore potential posture risks caused by tablet use. In this section we discuss our findings, present the limitations and highlight areas that would benefit from further investigation.

\subsection{Discussion}

Our findings show that research participants used their tablets in a way typical of other portable devices, i.e. in fixed positions that limit the range of possible postures (Heasman et al., 2000). Tablets were used primarily in sedentary positions with the device often held on a meeting table or a lap, confirming Young et al. (2012) findings. RULA identified the neck as one of the risk areas, which is not surprising, as neck discomfort is linked to keyboard use (Szeto et al., 2002). Since such discomfort can appear while using a physical keyboard on a workstation set up accordingly to health and safety guidelines, it is even more likely to occur when using a portable device with a screen that cannot be separated from the keyboard (European Commission, 2010) and which is not kept at an optimal height (Young et al., 2012).

The analysis also highlighted issues with wrist angles during typing, regardless of whether a cover was used to change the keyboard angle, which is in line with findings by Young et al. (2013). The more extended the wrist, the more flexed (or 'curled') fingers are (Nelson et al., 2000), which can increase discomfort, especially when users hold their fingers suspended in the air as was observed by Young et al. (2013) and during our study. Musculoskeletal risks could be reduced by using docking stations or external keyboards, although no studies have been conducted to evaluate tablet accessories. Gesturebased keyboards could also be considered: drawing shapes on a screen is less tiring to wrists and fingertips than typing as it does not require tapping force or holding hands in a fixed position, and users type one stroke per word instead of a tap per letter (Zhai \& Kristensson, 2003). However, it can be difficult to type without looking at the screen, neck discomfort would not be reduced and it is not clear what other muscles would be affected. Unfortunately, an ergonomic evaluation of a gesture-based keyboard on a tablet is also not yet available.

Glare can be a problem for tablet users due to the glossy screen, which was also mentioned by Hess and Jung (2012). Research participants reported a need to change their position to avoid reflections, and being unable to use their tablets outside on sunny days, which in both cases led to assuming poor posture and experiencing discomfort.
The portability of a tablet makes it difficult to ensure that the workstation is comfortable and follows health and safety guidelines, especially when existing regulations do not cover touch-screen tablets. However, while a standard regulating the design of a tablet would be beneficial, specific health and safety regulations may not be worthwhile, as it would not be possible to enforce them. Despite tablets having similar capabilities to laptops and posing similar risks, the context of use and the portability make them closer to smartphones, but there are no such smartphone regulations. Regulating tablet use would require defining good posture and, as observed during our research and reported by Young et al. (2012, 2013), maintaining good posture while using a tablet seems to be impossible. Enforcing the use of tablet accessories and external keyboards would also prove fruitless as users explicitly said they were not interested in such devices as they reduce portability.

\subsection{Limitations and future work}

Even though our research explored tablet use for office work and highlighted several problematic areas, a more rigorous postural analysis is needed. A study similar to research by Young et al. (2012, 2013), but focusing specifically on office work in the wild' (including commuting and use at home) could help to better understand the health implications. As tablet accessories may be used to reduce discomfort, an evaluation of external keyboards and docking stations is also needed to help understand their benefits and limitations, and suggest design improvements.

Nearly all of our research participants used Apple devices, and further research would benefit from considering other types of tablets, as different dimensions (size, weight) may cause varying levels of discomfort. As the questionnaire resulted in only 82 responses, a bigger study is needed to validate the findings. In addition, a longitudinal study could help to evaluate long-term effects of tablet use and mobile work on office workers and their posture.

As relevant health and safety guidelines do not exist, a detailed evaluation of existing regulations and standards would be beneficial to assess whether they can be adapted for tablet use and whether they are needed in the first place. Since employers are not able to control where tablets are used and therefore cannot be held fully responsible for users' health and safety, there may be no need for official tablet regulations as it would not be possible to enforce them. Moreover, as discomfort is cumulative and users use a number of portable devices, case law would not be able to prove the negative impact of a tablet. Minimising potential risks related to the use of tablets for office work is therefore a considerable future challenge for ergonomics. 


\section{REFERENCES}

Brand, J. L. (2008) Office ergonomics: A review of pertinent research and recent developments. In C. Carswell (Ed.), Reviews of human factors and ergonomics, Human Factors and Ergonomics Society, Santa Monica, CA

European Commission. (2010) The increasing use of portable computing and communication devices and its impact on the health of EU workers. Institute for Employment Studies.

Gartner. (2011) iPad and Beyond: The Media Tablet in Business.

http://www.gartner.com/resources/211700/211735/i pad_and_beyond_the_media_ta_211735.pdf

Geyer, M. \& Felske, F. (2011) Consumer Toy or Corporate Tool: The iPad Enters the Workplace, interactions, 18, 4, 45-49

Heasman, T., Brooks, A., \& Stewart, T. (2000) Health and safety of portable display screen equipment. HSE Books

Hess, S. \& Jung, J. (2012) Does the iPad add value to business environments? In Proc. CHI 2012, 335350, ACM Press

HSE (2003) Work with Display Screen Equipment: Health and Safety (Display Screen Equipment) Regulations 1992 as Amended by the Health and Safety (Miscellaneous Amendments) Regulations 2002: Guidance on Regulations. HSE Books

HSE (2006) Working with VDUs. Health and Safety Executive.

ISO (2007) ISO 9241-410:2007. Ergonomics of human-system interaction - Part 410: Physical input devices - Design criteria for products. Geneva: International Standards Organisation

Jafri, S. (2012) 5 Reasons the iPad may be Bad for Ergonomics. http://www.suite101.com/content/5reasons-the-ipad-may-be-bad-for-ergonomicsa222510 (retrieved 8/06/2013)

Lima, T. M., \& Coelho, D. A. (2011) Prevention of musculoskeletal disorders (MSDs) in office work: A case study, Work, 39, 4, 397-408

McAtamney, L., \& Corlett, N. (1993) RULA: a survey method for the investigation of work-related upper limb disorders, Applied Ergonomics, 24, 2, 91-99

Nelson, J. E., Treaster, D. E., \& Marras, W. S. (2000) Finger motion, wrist motion and tendon travel as a function of keyboard angles, Clinical
Biomechanics, 15, 7, 489-498

Nguyen, B. \& Chaparro, B. (2011) iPad Usage Patterns On-the-Go and at Work. Usability News, 13,2

OPA, (2012) A Portrait of Today's Tablet User, Wave II, Online Publishers Association \& Frank N. Magid Associates

Oborne, D. J. (1995) Ergonomics at Work. Human Factors in Design and Development. John Wiley \& Sons

\section{Ofcom (2012) Communications Market Report 2012 \\ http://stakeholders.ofcom.org.uk/binaries/research/ cmr/cmr12/CMR_UK_2012.pdf}

Pheasant, S., \& Haslegrave, C. M. (2006)

Bodyspace. Anthropometry, Ergonomics and the Design of Work (3rd ed.), Taylor \& Francis.

Stanton, N., Hedge, A., Brookhuis, K., Salas, E., \& Hendrick, H. (2005) Handbook of Human Factors and Ergonomics Methods. CRC Press LLC

Stawarz, K., Cox, A., Bird, J., Benedyk, R. (2013) "I'd sit at home and do work emails": How tablets affect the work-life balance of office workers, In Proc. CHI 2013, 1383-1388, ACM Press

Szeto, G. P., Straker, L., \& Raine, S. (2002) A field comparison of neck and shoulder postures in symptomatic and asymptomatic office workers, Applied Ergonomics, 33, 1, 75-84

Tessler, F. (2012) The hidden danger of touchscreens,

http://www.infoworld.com/t/laptops/the-hiddendanger-touchscreens-181774 (retrieved 8/06/2013)

UNISON (1998) Health and safety and the use of laptop computers. Guidelines for UNISON branches, safety representatives and stewards.

Young, J.G., Trudeau, M., Odell, D., Marineli, K., \& Dennerlein, J.T. (2012) Touch-screen tablet user configurations and case-supported tilt affect head and neck flexion angles, Work, 41, 1, 81-91

Young, J.G., Trudeau, M., Odell, D., Marinelli, K., \& Dennerlein, J.T. (2013) Wrist and shoulder posture and muscle activity during touch-screen tablet use: Effects on usage configuration, tablet type, and interacting hand, Work, 45, 1, 59-71

Zhai, S., \& Kristensson, P.-O. (2003) Shorthand writing on stylus keyboard, In: Proc. CHI 2003, 97104, ACM Press 